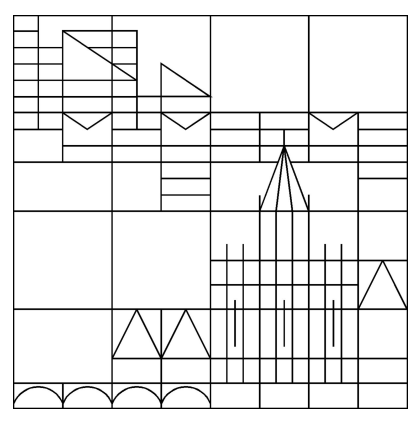

\title{
Nonlinear Well-Posedness and Rates of Decay in Thermoelasticity with Second Sound
}

\author{
Reinhard Racke \\ Ya-Guang Wang
}

Konstanzer Schriften in Mathematik und Informatik

Nr. 223, Januar 2007

ISSN $1430-3558$

Konstanzer Online-Publikations-System (KOPS)

URL: http://www.ub.uni-konstanz.de/kops/volltexte/2007/2278/

URN: http://nbn-resolving.de/urn:nbn:de:bsz:352-opus-22782

\footnotetext{
(C) Fachbereich Mathematik und Statistik

(C) Fachbereich Informatik und Informationswissenschaft

Universität Konstanz

Fach D 188, 78457 Konstanz, Germany

E-Mail: preprints@informatik.uni-konstanz.de

WWW: http://www.informatik.uni-konstanz.de/Schriften/
} 


\title{
Nonlinear Well-Posedness and Rates of Decay in Thermoelasticity with Second Sound
}

\author{
Reinhard RACKE and Ya-Guang WANG
}

Abstract: We consider the Cauchy problem in nonlinear thermoelasticity with second sound in one space dimension. Due to Cattaneo's law, replacing Fourier's law for heat conduction, the system is hyperbolic. We first investigate the local well-posedness as a strictly hyperbolic system, and then discuss the relation between energy estimates for non-symmetric hyperbolic systems and well-posedness. For the global small solution we describe the long time behavior and obtain decay rates of the $L^{2}$-norm.

\section{Introduction}

The nonlinear system of thermoelasticity in $\mathbb{R}^{n}$ where heat conduction is modeled by Cattaneo's law is given by differential equations for the displacement vector $u=u(t, x) \in \mathbb{R}^{n}$, the temperature $T=T(t, x)$, and the heat flux $q=q(t, x) \in \mathbb{R}^{n}, t \geq 0, x \in \mathbb{R}^{n}$. First we have the balance law of linear momentum in the absence of exterior forces,

$$
\rho u_{t t}-\operatorname{div} S=0
$$

where $\rho$ is the material density and assumed to be equal to one in the sequel; $S$ is the Piola-Kirchhoff stress tensor. Second we have the balance of energy in the absence of external heat supplies as

$$
\varepsilon_{t}-\operatorname{tr}\left\{S F_{t}\right\}+\operatorname{div} q=0
$$

where $\varepsilon$ denotes the internal energy, $\operatorname{tr}(\cdot)$ stands for the trace of a matrix, and $F$ is the deformation gradient,

$$
F=1+\nabla u
$$

Denoting by $\eta$ the specific entropy we get by

$$
\psi:=\varepsilon-T \eta
$$

the free energy. The first constitutive assumption, namely that $S, \varepsilon, \eta$ (and $\psi)$ are functions of $(\nabla u, \theta, q, \nabla \theta)$, where

$$
\theta=T-T_{0}
$$

denotes the temperature difference, $T_{0}>0$ the constant reference temperature - we shall assume homogeneity, i.e. no extra dependence on $x$ - is turned into the following relations after the second law of thermodynamics resp. the dissipation principle are applied (cp. $[1,2,8,15])$

$$
\psi=\psi(\nabla u, \theta, q)
$$

is independent of $\nabla \theta$, and

$$
\eta=\eta(\nabla u, \theta, q)=-\psi_{\theta}(\nabla u, \theta, q), \quad S=S(\nabla u, \theta, q)=\psi_{\nabla u}(\nabla u, \theta, q)
$$

\footnotetext{
${ }^{0}$ AMS subject classification: 74 F 05, 74 F 40, 35 B 40, 35 L 60. Keywords: Cauchy problems, strictly hyperbolic thermoelasticity, global existence,
} 
Using this, (1.2) turns into

$$
\left(\theta+T_{0}\right)\left\{a(\nabla u, \theta, q) \theta_{t}-\operatorname{tr}\left[S_{\theta} \cdot \nabla u_{t}\right]\right\}+\operatorname{div} q=b(\nabla u, \theta, q) q_{t}
$$

where

$$
a:=-\psi_{\theta \theta}, \quad b:=\left(\theta+T_{0}\right) \psi_{\theta q}-\psi_{q}
$$

For the heat flux, Cathaneo's law is assumed,

$$
\tau(\nabla u, \theta) q_{t}+q+k(\nabla u, \theta) \nabla \theta=0
$$

with the tensor of relaxation times $\tau$ and the heat conductivity tensor $k$. (1.6) is replacing the Fourier law

$$
q+k \nabla \theta=0
$$

formally arising from (1.6) by setting $\tau=0$, and then leading to the classical hyperbolic-parabolic-system for $(u, \theta)$ of thermoelasticity, cp. [8]. Here we use Cattaneo's law (1.6) avoiding the paradox of infinite propagation speed, and leading to a hyperbolic system (1.1), (1.4), (1.6) for $(u, \theta, q)$. We shall consider the one-dimensional Cauchy problem, i.e. (1.1), (1.4), (1.6) for $n=1$, together with initial conditions

$$
u(t=0)=u_{0}, u_{t}(t=0)=u_{1}, \theta(t=0)=\theta_{0}, q(t=0)=q_{0}
$$

In one space dimension (1.1), (1.4), (1.6) turn into the following, using $P:=\left(u_{x}, \theta, q\right)$ as abbreviation,

$$
\begin{gathered}
u_{t t}-a_{1}(P) u_{x x}+a_{2}(P) \theta_{x}+\sigma_{1}(P) q_{x}=0 \\
\theta_{t}+a_{3}(P) u_{t x}+a_{4}(P) q_{x}+\sigma_{2}(P) \theta_{x}+a_{5}(P) q=0 \\
q_{t}+a_{6}\left(u_{x}, \theta\right) \theta_{x}+a_{7}\left(u_{x}, \theta\right) q=0
\end{gathered}
$$

where

$$
\begin{gathered}
a_{1}:=S_{u_{x}}, a_{2}=-S_{\theta}, a_{3}=\frac{S_{\theta}}{\psi_{\theta \theta}}, \sigma_{1}=-S_{q}, a_{4}=\frac{-1}{\left(\theta+T_{0}\right) \psi_{\theta \theta}} \\
a_{5}=\frac{-b}{\tau\left(\theta+T_{0}\right) \psi_{\theta \theta}}, a_{6}=\frac{k}{\tau}, a_{7}=\frac{1}{\tau}, \sigma_{2}=\frac{-b k}{\tau\left(\theta+T_{0}\right) \psi_{\theta \theta}}
\end{gathered}
$$

where we assume $|\theta|<T_{0}$ which will be a posteriori justified for the global small solution. The precise assumptions on the coefficients will be given in section 2, they are the natural ones from the physical model like $a_{1}>0$, and so on.

First we shall be interested in the local well-posedness for (1.8) - (1.11), for data $\left(u_{x}, u_{t}, \theta, q\right)(t=0)$ in $W^{s, 2}\left(\mathbb{R}^{1}\right)$, the usual Sobolev space with $s>\frac{3}{2}$. A local existence result was stated in the work of Tarabek [19], just with a hint to the paper of Hughes, Kato and Marsden [7] which needs some explanation, at least in the way we present a proof below.

For initial-boundary value problems in bounded reference configurations (intervals) $\Omega \subset \mathbb{R}^{1}$, local existence theorems were stated in [15] and by Messaoudi and Said-Houari [12]; these are justified a posteriori by the possibility of proving appropriate (exponential type) energy estimates there.

We shall demonstrate here that the system (1.9) - (1.11) is strictly hyperbolic provided $\left|\sigma_{1}\right|$ is small enough for which, in turn, $\left|\left(u_{x}, \theta, q\right)\right|$ being small is sufficient because we have

$$
\sigma_{1}(0,0,0)=0 .
$$

Observe that $\sigma_{1} \neq 0$ perturbs the more or less given symmetric-hyperbolic structure of (1.9) - (1.11) when $\sigma_{1}=0$. The term $\sigma_{1} q_{x}$ (actually: (1.9)) not appearing for pure heat conductions allowed Coleman, 
Hrusa and Owen [3] to conclude the local well-posedness from the symmetric-hyperbolic setting.

The possibility of proving local existence for nonlinear hyperbolic systems is closely related to the possibility of proving energy estimates in $H^{s}$-norms, which can be guaranteed for symmetrizable hyperbolic systems, cp. the work of Taylor [20, Chapter 5], or Kato [9, 10].

In Section 2 we shall prove the strict hyperbolicity of (1.9) - (1.11), and in Section 3 we look at a (linear) model example that will illustrate the interplay between energy estimates and well-posedness, giving insight into possible future extensions to higher space dimensions.

Having given the global solution for small data $\left(u_{x}, u_{t}, \theta, q\right)(t=0)$ in $H^{2}$ from Tarabek [19], we shall describe in Section 4 the asymptotic behavior of $V(t) \equiv\left(u_{x}, u_{t}, \theta, q\right)(t)$ as time $t \rightarrow \infty$.

For the corresponding linearized system Yang and Wang [24], also W. Wang and Z. Wang [21], gave decay rates for $L^{q}$-norms of $V(t)$ (cp. also Wang and Yang [23] in three space dimensions).

For the nonlinear system, based on the experience with the classical system and Fourier's law, see $[4,5,6,8]$, one has to expect the formation of singularities for large data, while small data should lead to global solutions and a polynomial decay of $L^{q}$-norms, see [8] for a survey. The propagation of singularities has been discussed by the authors both for Fourier's and for Cattaneo's law, cp. [16, 17, 18, 22]. The considerations for the classical (Fourier) case use special divergence forms of the nonlinearities, cp. [8], which are not given for the general Cattaneo law. Therefore, we restrict ourselves to a linear Cattaneo law

$$
\tau_{0} q_{t}+q+\kappa \theta_{x}=0
$$

where $\tau_{0}, \kappa>0$ are constants. As a result we shall obtain that the $L^{2}$-norm of $V(t)$ decays of order $(1+t)^{-\frac{1}{4}}$.

We use standard Sobolev spaces $W^{s, p}(\mathbb{R}), s \in \mathbb{N}_{0}, 1 \leq p \leq \infty$, with norm $\|\cdot\|_{s, p}$, and $\|\cdot\|_{p}:=\|\cdot\|_{0, p}$ in $L^{p}(\mathbb{R})$.

\section{Local well-posedness}

Here we consider the Cauchy problem (1.8) - (1.11), i.e.

$$
\begin{array}{r}
u_{t t}-a_{1}(P) u_{x x}+a_{2}(P) \theta_{x}+\sigma_{1}(P) q_{x}=0 \\
\theta_{t}+a_{3}(P) u_{t x}+a_{4}(P) q_{x}+\sigma_{2}(P) \theta_{x}+a_{5}(P) q=0 \\
q_{t}+a_{6}\left(u_{x}, \theta\right) \theta_{x}+a_{7}\left(u_{x}, \theta\right) q=0 \\
u(0, x)=u_{0}(x), u_{t}(0, x)=u_{1}(x), \theta(0, x)=\theta_{0}(x), q(0, x)=q_{0}(x), x \in \mathbb{R}
\end{array}
$$

where

$$
P=\left(u_{x}, \theta, q\right)
$$

and the coefficients

$$
\begin{gathered}
a_{1}=S_{u_{x}}, a_{2}=-S_{\theta}, a_{3}=\frac{S_{\theta}}{\psi_{\theta \theta}}, \sigma_{1}=-S_{q}, a_{4}=\frac{-1}{\left(\theta+T_{0}\right) \psi_{\theta \theta}} \\
a_{5}=\frac{-b}{\tau\left(\theta+T_{0}\right) \psi_{\theta \theta}}, a_{6}=\frac{k}{\tau}, a_{7}=\frac{1}{\tau}, \sigma_{2}=\frac{-b k}{\tau\left(\theta+T_{0}\right) \psi_{\theta \theta}} \\
\sigma_{1}(0)=0, \sigma_{2}(0)=0, a_{5}(0)=0
\end{gathered}
$$

satisfy the physically natural assumptions that $a_{1}, a_{2} \cdot a_{3}, a_{4} \cdot a_{6}, \tau, k$ are positive, that is, there are $\underline{a}_{1}^{0}, \underline{a}_{23}^{0}, \underline{a}_{46}^{0}, \tau^{0}, k^{0}>0$ such that for all $P=\left(u_{x}, \theta, q\right)$ we have

$$
a_{1}(P) \geq \underline{a}_{1}^{0}, \quad a_{2}(P) a_{3}(P) \geq \underline{a}_{23}^{0}, \quad a_{4}(P) a_{6}(P) \geq \underline{a}_{46}^{0}
$$




$$
\tau\left(u_{x}, \theta\right) \geq \tau^{0}, k\left(u_{x}, \theta\right) \geq k^{0} .
$$

In view of the later global small data result we remark that it would be sufficient to require (2.8), (2.9) for small $|P|$; moreover, we assume $|\theta|<T_{0}$.

Transforming (2.1) - (2.4) into a first-order system for

$$
\begin{gathered}
V:=\left(u_{x}, u_{t}, \theta, q\right)^{\prime} \\
V_{t}+\underbrace{\left(\begin{array}{cccc}
0 & -1 & 0 & 0 \\
-a_{1} & 0 & a_{2} & \sigma_{1} \\
0 & a_{3} & \sigma_{2} & a_{4} \\
0 & 0 & a_{6} & 0
\end{array}\right)}_{=: A(V)} \partial_{x} V+\underbrace{\left(\begin{array}{cccc}
0 & 0 & 0 & 0 \\
0 & 0 & 0 & 0 \\
0 & 0 & 0 & a_{5} \\
0 & 0 & 0 & a_{7}
\end{array}\right)}_{=: B(V)} V=0 \\
V(t=0)=V_{0}:=\left(u_{0, x}, u_{1}, \theta_{0}, q_{0}\right)^{\prime}
\end{gathered}
$$

we notice that if $\sigma_{1}=0$ then (2.10) could be turned into a symmetric-hyperbolic system, cp. [3] for the case of pure heat conduction with second sound, and then the local well-posedness would follow immediately.

Here we shall show that (2.10) is strictly hyperbolic if $\left|\sigma_{1}\right|$ is small enough which is satisfied if $|V|$ is small enough since $\sigma_{1}(0)=0$ by $(2.7)$.

Lemma 2.1 There is $\delta>0$ such that for all $P$ with $|P|<\delta$ (2.10) is strictly hyperbolic.

Proof: We have to show that the four eigenvalues of $A(V)$ are real and distinct. These eigenvalues are the zeros of

$$
\begin{aligned}
h_{\sigma}(\lambda) & :=\operatorname{det}\left(\lambda \mathrm{I}_{\mathbb{C}^{4}}-A(V)\right) \\
& =\lambda^{4}-\sigma_{2} \lambda^{3}-\left(a_{2} a_{3}+a_{4} a_{6}+a_{1}\right) \lambda^{2}-\left(a_{3} a_{6} \sigma_{1}-a_{1} \sigma_{2}\right) \lambda+a_{1} a_{4} a_{6}
\end{aligned}
$$

where $\sigma:=\left(\sigma_{1}, \sigma_{2}\right)$. We have

$$
h_{\sigma}( \pm \infty)=+\infty, \quad h_{\sigma}(0)=a_{1} a_{4} a_{6}>0
$$

by assumption (2.8).

Let

$$
\mu_{ \pm}:= \pm \sqrt{a_{1}}
$$

then

$$
\mu_{-}<0<\mu_{+}
$$

and

$$
h_{\sigma}\left(\mu_{ \pm}\right)=-\sqrt{a_{1}}\left(\sqrt{a_{1}} a_{2} a_{3} \pm \sigma_{1} a_{3} a_{6}\right) \equiv-\sqrt{a_{1}} \cdot Q
$$

Assuming without loss of generality

$$
\exists K>0:|P| \leq K
$$

we conclude that there exist $\bar{a}_{3}^{0}, \bar{a}_{6}^{0}>0$ such that for all $P$ with $|P| \leq K$ we have

$$
\left|a_{3}(P)\right| \leq \bar{a}_{3}^{0}, \quad\left|\bar{a}_{6}(P)\right| \leq \bar{a}_{6}^{0}
$$


which implies, using (2.8),

$$
\begin{aligned}
Q & \geq \sqrt{a_{1}} a_{2} a_{3}-\left|\sigma_{1}\right|\left|a_{3}\right|\left|a_{6}\right| \\
& \geq \sqrt{\underline{a}_{1}^{0}} \underline{a}_{23}^{0}-\left|\sigma_{1}\right| \bar{a}_{3}^{0} \bar{a}_{6}^{0} \\
& \geq \frac{\sqrt{\underline{a}_{1}^{0}} \underline{a}_{23}^{0}}{2}
\end{aligned}
$$

if

$$
\left|\sigma_{1}\right| \leq \sigma^{0}:=\frac{\sqrt{\underline{a}_{1}^{0}} \underline{a}_{23}^{0}}{2 \bar{a}_{3}^{0} \bar{a}_{6}^{0}}
$$

which, by $(2.7)$, is satisfied if

$$
|P|=\left|\left(u_{x}, \theta, q\right)\right| \leq \delta=\delta\left(\sigma^{0}\right)
$$

By (2.14), (2.17), (2.19) we thus have

$$
\exists \delta>0 \forall P,|P| \leq \delta: \quad h_{\sigma}( \pm \mu)<0
$$

Then the combination of (2.12), (2.20), observing (2.13) yields that $h_{\sigma}$ has four distinct real zeros if $|P| \leq \delta=\delta\left(\sigma^{0}\right)$.

Q.e.d.

We remark that with the special choice of $\mu_{ \pm}$it was possible to give a detailed estimate for $\sigma^{0}$ in (2.18), depending on the coefficient functions, hence also an estimate for $\delta$ appearing in Lemma 2.1 is possible depending on $\sigma_{1}$, cp. (2.19).

The strict hyperbolicity of (2.10) now implies the local well-posedness, see e.g. [20, chapter 5], it also implies that (2.10) is symmetrizable. Thus we conclude

Theorem 2.2 Let $s \geq 2$. Then there is $\delta>0$ such that for data $V_{0} \in W^{s, 2}(\mathbb{R})$ with $\left\|V_{0}\right\|_{s, 2}<\delta$ there is a unique local solution $V$ to (2.10), (2.11) in some time interval $[0, T]$ with

$$
V \in C^{0}\left([0, T], W^{s, 2}(\mathbb{R})\right) \cap C^{1}\left([0, T], W^{s-1,2}(\mathbb{R})\right),
$$

and $T=T\left(\left\|V_{0}\right\|_{s, 2}\right)>0$. Moreover, $\delta$ is determined by (2.18), (2.19).

In [19] a local well-posedness result is stated with a hint to the work of Hughes, Kato and Marsden [7]; we believe that additional comments there would have been necessary and, in turn, presented our results above.

\section{On the relation energy estimates - well-posedness}

It is well known that the possibility of proving a local well-posedness result in $W^{s, 2}$-spaces - for rather general (hyperbolic) evolution equations - is strongly connected to proving energy estimates in the $W^{s, 2}$-norm, and in proving the well-posedness plus energy estimates for associated linear systems, cp. Taylor's book [20] and the work of Kato [9, 10].

We shall illustrate this with a linear model example associated to our nonlinear system (2.1) - (2.4).

This will not only give insight into the relation " energy estimate - well-posedness " but might be also a starting point for proving the local well-posedness in more than one space dimension, where we have to expect multiple eigenvalues (because of the elastic part) and hence then could not argue with strict 
hyperbolicity.

The model problem is, cp. (2.1) - (2.4), the following linear Cauchy problem,

$$
\begin{aligned}
u_{t t}-u_{x x}+\theta_{x}+\sigma_{1} q_{x} & =0 \\
\theta_{t}+u_{t x}+q_{x}+\sigma_{2} \theta_{x} & =0 \\
q_{t}+\theta_{x}+q & =0 \\
u(t=0)=u_{0}, u_{t}(t=0)=u_{1}, \theta(t=0) & =\theta_{0}, q(t=0)=q_{0}
\end{aligned}
$$

where $\sigma_{1}, \sigma_{2}$ are real constants. Defining $V:=\left(u_{x}, u_{t}, \theta, q\right)^{\prime}$ we transform (3.1) - (3.4) into

$$
V_{t}+A V=0, \quad V(t=0)=V^{0}:=\left(u_{0, x}, u_{1}, \theta_{0}, q_{0}\right)^{\prime}
$$

with, formally,

$$
A=\left(\begin{array}{cccc}
0 & -1 & 0 & 0 \\
-1 & 0 & 1 & \sigma_{1} \\
0 & 1 & \sigma_{2} & 1 \\
0 & 0 & 1 & 0
\end{array}\right) \partial_{x}+\left(\begin{array}{cccc}
0 & 0 & 0 & 0 \\
0 & 0 & 0 & 0 \\
0 & 0 & 0 & 0 \\
0 & 0 & 0 & 1
\end{array}\right)
$$

We shall prove that $A$, as operator

$$
A: D(A):=\left(W^{1,2}(\mathbb{R})\right)^{4} \subset\left(L^{2}(\mathbb{R})\right)^{4} \equiv \mathcal{H} \rightarrow \mathcal{H}
$$

generates a $C_{0}$-semigroup in $\mathcal{H}$, where the inner product in $\mathcal{H}$ will be a modified $L^{2}$ inner product. This modification will be suggested by the following energy estimate for a (time-dependent) solution to (3.1) - (3.4), hence demonstrating the connections, energy estimates - well-posedness - generator property, clearly.

So, assume that we have an appropriate solution $(u, \theta, q)$ to (3.1) - (3.4). Then multiply (3.1) by $u_{t}$, (3.2) by $\theta$, and (3.3) by $q$, respectively, and intergrate in $L^{2}(\mathbb{R})$. We obtain

$$
\frac{d}{d t}\left\{\frac{1}{2} \int_{\mathbb{R}}\left(u_{t}^{2}+u_{x}^{2}+\theta^{2}+q^{2}\right) d x\right\}+\int_{\mathbb{R}} q^{2} d x+\sigma_{1} \int_{\mathbb{R}} q_{x} u_{t} d x=0
$$

Partially integrating and using the differential equation (3.2) we get

$$
\begin{aligned}
\sigma_{1} \int_{\mathbb{R}} q_{x} u_{t} d x & =-\sigma_{1} \int_{\mathbb{R}} q u_{t x} d x=\sigma_{1} \int_{\mathbb{R}} q \theta_{t} d x+\sigma_{1} \int_{\mathbb{R}} q q_{x} d x+\sigma_{1} \sigma_{2} \int_{\mathbb{R}} q \theta_{x} d x \\
& =\sigma_{1} \int_{\mathbb{R}} q \theta_{t} d x+\sigma_{1} \sigma_{2} \int_{\mathbb{R}} q \theta_{x} d x \\
& =\frac{d}{d t}\left\{\sigma_{1} \int_{\mathbb{R}} q \theta d x\right\}-\sigma_{1} \int_{\mathbb{R}} q_{t} \theta d x+\sigma_{1} \sigma_{2} \int_{\mathbb{R}} q \theta_{x} d x
\end{aligned}
$$

Now using the differential equation (3.3) we conclude

$$
\begin{aligned}
\sigma_{1} \int_{\mathbb{R}} q_{x} u_{t} d x= & \frac{d}{d t}\left\{\sigma_{1} \int_{\mathbb{R}} q \theta d x\right\}+\sigma_{1} \int_{\mathbb{R}} \theta_{x} \theta d x+\sigma_{1} \int_{\mathbb{R}} q \theta d x \\
& -\sigma_{1} \sigma_{2} \frac{d}{d t}\left\{\frac{1}{2} \int_{\mathbb{R}} q^{2} d x\right\}-\sigma_{1} \sigma_{2} \int_{\mathbb{R}} q^{2} d x \\
= & \frac{d}{d t}\left\{\frac{1}{2} \int_{\mathbb{R}}\left(-\sigma_{1} \sigma_{2} q^{2}+2 \sigma_{1} q \theta\right) d x\right\}+\sigma_{1} \int_{\mathbb{R}} q \theta d x-\sigma_{1} \sigma_{2} \int_{\mathbb{R}} q^{2} d x
\end{aligned}
$$


Define

$$
E(t):=\frac{1}{2} \int_{\mathbb{R}}\left(u_{t}^{2}+u_{x}^{2}+\theta^{2}+\left(1-\sigma_{1} \sigma_{2}\right) q^{2}+2 \sigma_{1} q \theta\right) d x
$$

which is a positively definite quadratic form if

$$
\sigma_{1} \sigma_{2}+\sigma_{1}^{2}<1
$$

Combining (3.7), (3.8) we get

$$
\frac{d}{d t} E(t)=-\left(1-\sigma_{1} \sigma_{2}\right) \int_{\mathbb{R}} q^{2} d x-\int_{\mathbb{R}} \sigma_{1} q \theta d x \leq c E(t)
$$

with a positive constant $c=c\left(\sigma_{1}, \sigma_{2}\right)$.

From (3.11), we conclude

$$
0 \leq E(t) \leq \mathrm{e}^{c t} E(0)
$$

that is, we have a first a priori estimate. In the present linear case we could get the corresponding a priori estimate for $(u, \theta, q)$ in $W^{s, 2}$-norms by differentiating $(3.1)$ - (3.3), with respect to $x$. Now we turn to the evolution system (3.5) for $V$. Motivated by (3.12) and the definition of $E(t)$ in $(3.9)$ we choose the following inner product in $\mathcal{H}=\left(L^{2}(\mathbb{R})\right)^{4}$ :

$$
\langle V, W\rangle_{\mathcal{H}}:=\int_{\mathbb{R}}\left(V^{1} W^{1}+V^{2} W^{2}+V^{3} W^{3}+\left(1-\sigma_{1} \sigma_{2}\right) V^{4} W^{4}+\sigma_{1} V^{3} W^{4}+\sigma_{1} V^{4} W^{3}\right) d x
$$

with associated norm $\|\cdot\|_{\mathcal{H}}$.

By assumption (3.10), it is indeed an inner product and equivalent to the standard inner product in $L^{2}(\mathbb{R})$. For a solution $V=V(t)$ we thus well have

$$
E(t)=\frac{1}{2}\|V(t)\|_{\mathcal{H}}^{2}
$$

Because of the expected energy estimate (3.11) we expect

$$
\left|\langle A V(t), V(t)\rangle_{\mathcal{H}}\right| \leq \frac{c}{2}\|V(t)\|_{\mathcal{H}}^{2}
$$

to hold true. Requiring further $4 \sigma_{1}^{2}+3 \sigma_{1} \sigma_{2} \leq 3$, we can prove

Lemma 3.1 For $V \in D(A)$ we have

$$
\left|\langle A V, V\rangle_{\mathcal{H}}\right| \leq \frac{3}{2}\|V\|_{\mathcal{H}}^{2}
$$

ProOF:

$$
\begin{aligned}
\langle A V, V\rangle_{\mathcal{H}} & =-\int_{\mathbb{R}} V_{x}^{2} V^{1} d x-\int_{\mathbb{R}}\left(V_{x}^{1}-V_{x}^{3}-\sigma_{1} V_{x}^{4}\right) V^{2} d x \\
& +\int_{\mathbb{R}}\left(V_{x}^{2}+\sigma_{2} V_{x}^{3}+V_{x}^{4}\right) V^{3} d x+\left(1-\sigma_{1} \sigma_{2}\right) \int_{\mathbb{R}}\left(V_{x}^{3}+V^{4}\right) V^{4} d x \\
& +\sigma_{1} \int_{\mathbb{R}}\left(V_{x}^{2}+\sigma_{2} V_{x}^{3}+V_{x}^{4}\right) V^{4} d x+\sigma_{1} \int_{\mathbb{R}}\left(V_{x}^{3}+V^{4}\right) V^{3} d x \\
& =\left(1-\sigma_{1} \sigma_{2}\right) \int_{\mathbb{R}}\left|V^{4}\right|^{2} d x+\sigma_{1} \int_{\mathbb{R}} V^{3} V^{4} d x
\end{aligned}
$$


This implies

$$
\left|\langle A V, V\rangle_{\mathcal{H}}\right| \leq \frac{3}{2}\|V\|_{\mathcal{H}}^{2}
$$

when $4 \sigma_{1}^{2}+3 \sigma_{1} \sigma_{2} \leq 3$. Q.e.d.

As a consequence we have for $d>\frac{3}{2}$ and $d_{1}:=d-\frac{3}{2}$ :

$$
\langle(A-d) V, V\rangle_{\mathcal{H}} \leq-d_{1}\|V\|_{\mathcal{H}}^{2}
$$

Theorem 3.2 The operator $A$ generates a $C_{0}$-semigroup if $3 \sigma_{1} \sigma_{2}+4 \sigma_{1}^{2} \leq 3$.

Proof: By the previous lemma and (3.13) it is sufficient to show that the range of $(A-d)$ satisfies

$$
R(A-d)=\mathcal{H}
$$

if $d>\frac{3}{2}$ is sufficiently large. Since $R(A-d)=\overline{R(A-d)}$ the orthogonal decomposition

$$
\mathcal{H}=R(A-d) \oplus N\left(A^{*}-d\right)
$$

holds, where $A^{*}$ denotes the adjoint of $A$ in $\mathcal{H}$, and $N$ denotes the nullspace. Thus it is, observing (3.13), sufficient to show that the domains of $A$ and $A^{*}$ satisfy

$$
D(A)=D\left(A^{*}\right)
$$

(a) Fix $V \in D(A)$, and let $\varphi \in D(A)$ be arbitrary. Then

$$
\begin{aligned}
\langle A \varphi, V\rangle_{\mathcal{H}}= & -\int_{\mathbb{R}} \varphi_{x}^{2} V^{1} d x-\int_{\mathbb{R}}\left(\varphi_{x}^{1}-\varphi_{x}^{3}-\sigma_{1} \varphi_{x}^{4}\right) V^{2} d x \\
& +\int_{\mathbb{R}}\left(\varphi_{x}^{2}+\sigma_{2} \varphi_{x}^{3}+\varphi_{x}^{4}\right) V^{3} d x+\left(1-\sigma_{1} \sigma_{2}\right) \int_{\mathbb{R}}\left(\varphi_{x}^{3}+\varphi^{4}\right) V^{4} d x \\
& +\sigma_{1} \int_{\mathbb{R}}\left(\varphi_{x}^{2}+\sigma_{2} \varphi_{x}^{3}+\varphi_{x}^{4}\right) V^{4} d x+\sigma_{1} \int_{\mathbb{R}}\left(\varphi_{x}^{3}+\varphi^{4}\right) V^{3} d x \\
= & \int_{\mathbb{R}} \varphi^{1} V_{x}^{2} d x+\int_{\mathbb{R}} \varphi^{2}\left(V_{x}^{1}-V_{x}^{3}-\sigma_{1} V_{x}^{4}\right) d x \\
& +\int_{\mathbb{R}} \varphi^{3} \underbrace{\left(-V_{x}^{2}-\sigma_{2} V_{x}^{3}-V_{x}^{4}-\sigma_{1} V_{x}^{3}\right)}_{=: f_{2}} d x \\
& +\int_{\mathbb{R}} \varphi^{4} \underbrace{\left(-\sigma_{1} V_{x}^{2}-V_{x}^{3}+\left(1-\sigma_{1} \sigma_{2}\right) V^{4}-\sigma_{1} V_{x}^{4}+\sigma_{1} V^{3}\right)} d x
\end{aligned}
$$

This should equal

$$
\langle\varphi, F\rangle_{\mathcal{H}} \quad\left(=\left\langle\varphi, A^{*} V\right\rangle_{\mathcal{H}} \text { then }\right)
$$

for some $F \in \mathcal{H}$. That is, we can choose

$$
F^{1}:=V_{x}^{2}, \quad F^{2}:=V_{x}^{1}-V_{x}^{3}-\sigma_{1} V_{x}^{4}
$$

and shall determine $F^{3}, F^{4}$ as solutions to

$$
\begin{aligned}
F^{3}+\sigma_{1} F^{4} & =f_{1} \\
\sigma_{1} F^{3}+\left(1-\sigma_{1} \sigma_{2}\right) F^{4} & =f_{2}
\end{aligned}
$$


Since $\sigma_{1}^{2}+\sigma_{1} \sigma_{2} \neq 1$ the system (3.18), (3.19) is uniquely solvable,

$$
\begin{aligned}
F^{3}= & \frac{1}{1-\left(\sigma_{1}^{2}+\sigma_{1} \sigma_{2}\right)}\left(\left(\sigma_{1}^{2}+\sigma_{1} \sigma_{2}-1\right) V_{x}^{2}+\left(\sigma_{1}^{2}+\sigma_{1} \sigma_{2}-1\right) V_{x}^{4}+\right. \\
& \left.\left(\sigma_{1}^{2} \sigma_{2}+\sigma_{1} \sigma_{2}^{2}-\sigma_{2}\right) V_{x}^{3}-\sigma_{1}^{2} V^{3}-\left(\sigma_{1}-\sigma_{1}^{2} \sigma_{2}\right) V^{4}\right) \\
F^{4}= & \frac{1}{1-\left(\sigma_{1}^{2}+\sigma_{1} \sigma_{2}\right)}\left(\sigma_{1} V^{3}+\left(1-\sigma_{1} \sigma_{2}\right) V^{4}-\left(1-\left(\sigma_{1}^{2}+\sigma_{1} \sigma_{2}\right)\right) V_{x}^{3}\right)
\end{aligned}
$$

Thus we have proved

$$
D(A) \subset D\left(A^{*}\right)
$$

and obtained a formal representation of $A^{*}$ as

$$
A^{*}=\left(\begin{array}{cccc}
0 & \partial_{x} & 0 & 0 \\
\partial_{x} & 0 & -\partial_{x} & -\sigma_{1} \partial_{x} \\
0 & -\partial_{x} & \left(\frac{\sigma_{1}^{2} \sigma_{2}+\sigma_{1} \sigma_{2}^{2}-\sigma_{2}}{1-\left(\sigma_{1}^{2}+\sigma_{1} \sigma_{2}\right)} \partial_{x}-\frac{\sigma_{1}^{2}}{1-\left(\sigma_{1}^{2}+\sigma_{1} \sigma_{2}\right)}\right) & \left(-\partial_{x}-\frac{\sigma_{1}-\sigma_{1}^{2} \sigma_{2}}{1-\left(\sigma_{1}^{2}+\sigma_{1} \sigma_{2}\right)}\right) \\
0 & 0 & \left(-\partial_{x}+\frac{\sigma_{1}}{1-\left(\sigma_{1}^{2}+\sigma_{1} \sigma_{2}\right)}\right) & \frac{1-\sigma_{1} \sigma_{2}}{1-\left(\sigma_{1}^{2}+\sigma_{1} \sigma_{2}\right)}
\end{array}\right)
$$

(b) Now fix $V \in D\left(A^{*}\right)$. Then there is $F \in \mathcal{H}$ such that

$$
\forall \varphi \in D(A):\langle A \varphi, V\rangle_{\mathcal{H}}=\langle\varphi, F\rangle_{\mathcal{H}}
$$

(i) Taking $\varphi=\left(\varphi^{1}, 0,0,0\right)^{\prime}, \varphi^{1} \in C_{0}^{\infty}(\mathbb{R})$, then from (3.24)

$$
-\int_{\mathbb{R}} \varphi_{x}^{1} V^{2} d x=\int_{\mathbb{R}} \varphi^{1} F^{1}
$$

hence

$$
V^{2} \in W^{1,2}(\mathbb{R})
$$

(ii) Taking $\varphi=\left(0, \varphi^{2}, 0,0,\right)^{\prime}, \varphi^{2} \in C_{0}^{\infty}(\mathbb{R})$, then from (3.24)

$$
-\int_{\mathbb{R}} \varphi_{x}^{2} V^{1} d x+\int_{\mathbb{R}} \varphi_{x}^{2} V^{3} d x+\int_{\mathbb{R}} \varphi_{x}^{2} \sigma_{1} V^{4} d x=\int_{\mathbb{R}} \varphi^{2} F^{2} d x
$$

implying

$$
-V^{1}+V^{3}+\sigma_{1} V^{4} \in W^{1,2}(\mathbb{R})
$$

(iii) Taking $\varphi=\left(0,0, \varphi^{3}, 0,\right)^{\prime}, \varphi^{3} \in C_{0}^{\infty}(\mathbb{R})$, then from (3.24)

$$
\int_{\mathbb{R}} \varphi_{x}^{3} V^{2} d x+\sigma_{2} \int_{\mathbb{R}} \varphi_{x}^{3} V^{3} d x+\int_{\mathbb{R}} \varphi_{x}^{3} V^{4} d x+\int_{\mathbb{R}} \varphi_{x}^{3} \sigma_{1} V^{3} d x=\int_{\mathbb{R}} \varphi^{3}\left(F^{3}+\sigma_{1} F^{4}\right) d x
$$

implying, using (3.18),

$$
V^{4}+\left(\sigma_{1}+\sigma_{2}\right) V^{3} \in W^{1,2}(\mathbb{R})
$$

(iv) Taking $\varphi=\left(0,0,0, \varphi^{4}\right), \varphi^{4} \in C_{0}^{\infty}(\mathbb{R})$, then from (3.24)

$$
\begin{gathered}
\int_{\mathbb{R}} \sigma_{1} \varphi_{x}^{4} V^{2} d x+\int_{\mathbb{R}} \varphi_{x}^{4} V^{3} d x+\left(1-\sigma_{1} \sigma_{2}\right) \int_{\mathbb{R}} \varphi^{4} V^{4} d x+\sigma_{1} \int_{\mathbb{R}} \varphi_{x}^{4} V^{4} d x+\sigma_{1} \int_{\mathbb{R}} \varphi^{4} V^{3} d x \\
=\int_{\mathbb{R}} \varphi^{4}\left(\left(1-\sigma_{1} \sigma_{2}\right) F^{4}+\sigma_{1} F^{3}\right) d x
\end{gathered}
$$

implying

$$
V^{3}+\sigma_{1} V^{4} \in W^{1,2}(\mathbb{R})
$$


(3.27), (3.28) determine $V^{3}, V^{4}$ (pointwise first) since $\sigma_{1}^{2}+\sigma_{1} \sigma_{2} \neq 1$, cp. (3.18) - (3.21), and we conclude

$$
V^{3}, V^{4} \in W^{1,2}(\mathbb{R})
$$

This combined with (3.26) yields

$$
V^{1} \in W^{1,2}(\mathbb{R})
$$

By (3.25), (3.29), (3.30) we have proved

$$
V \in\left(W^{1,2}(\mathbb{R})\right)^{4}=D(A)
$$

that is

$$
D\left(A^{*}\right) \subset D(A)
$$

(3.22), (3.31) yield (3.15) hence (3.14), and the proof of Theorem 3.2 is finished.

Q.E.D.

Thus (3.5), respectively (3.1) - (3.4), is well-posed,

$$
V(t)=e^{-A t} V_{0}
$$

and the connections illustrated in this section might be useful for the consideration of the local wellposedness in several space dimensions.

\section{Decay rates of global solutions}

Having settled the local well-posedness of the nonlinear system (2.10) - (2.11) in Theorem 2.2, we can now take the global existence theorem from Tarabek([19]), yielding

Theorem 4.1 ([19]) Let $s \geq 2$. Then there is $\delta>0$ such that for data $V_{0} \in W^{s, 2}(\mathbb{R})$ with $\left\|V_{0}\right\|_{s, 2}<\delta$ there exists a unique global solution $V$ to (2.10), (2.11) satisfying

$$
V \in C^{0}\left([0, \infty), W^{s, 2}(\mathbb{R})\right) \cap C^{1}\left([0, \infty), W^{s-1,2}(\mathbb{R})\right)
$$

and

$$
\exists K>0, \forall t \geq 0:\|V(t)\|_{s, 2} \leq K\left\|V_{0}\right\|_{s, 2} .
$$

The last estimate is implicitly contained in [19]. There the case $s=2$ is studied but standard techniques yield $s \geq 2$ arbitrary. Tarabek also proved that $\|V(t)\|_{1, \infty}$ and $\|\nabla V(t)\|_{2}$ tend to zero as $t \rightarrow \infty$. But the rates of decay were not given. Here we shall give polynomial decay rates for the solution. This will be based on the knowledge of the asymptotic behavior of solutions to the corresponding linearized system which we can take from the work of Yang \& Wang [23] and of W. Wang \& Z. Wang [21]. Then suitable a priori estimates have to be proved in the spirit of the method described in general in [14], and already used in classical (hyperbolic-parabolic) thermoelasticity now connected to Cattaneo's law.

We can rewrite the differential equations (1.9) - (1.11) as follows

$$
\begin{aligned}
u_{t t}-\alpha u_{x x}+\beta \theta_{x} & =h_{1} \\
\theta_{t}+\gamma q_{x}+\delta u_{t x} & =h_{2} \\
\tau_{0} q_{t}+q+\kappa \theta_{x} & =h_{3}
\end{aligned}
$$


where (cp. (1.12), (1.13))

$$
\begin{aligned}
\alpha & :=S_{u_{x}}(0,0,0), \quad \beta:=-S_{\theta}(0,0,0), \quad \gamma:=a_{4}(0,0,0) \\
\delta & :=a_{3}(0,0,0), \quad \kappa:=\tau_{0} a_{6}(0,0,0), \quad \tau_{0}:=\tau(0,0) \\
h_{1} & :=\left(S\left(u_{x}, \theta, q\right)-\alpha u_{x}+\beta \theta\right)_{x} \\
h_{2} & :=\left(\gamma-a_{4}\right) q_{x}+\left(\delta-a_{3}\right) u_{t x}-\sigma_{2} \theta_{x}-a_{5} q \\
h_{3} & :=\tau_{0}\left\{\left(\frac{1}{\tau_{0}}-\frac{1}{\tau}\right) q+\left(\frac{\kappa}{\tau_{0}}-\frac{k}{\tau}\right) \theta_{x}\right\} .
\end{aligned}
$$

One knows

$$
\sigma_{1}(0,0,0)=0, \quad \sigma_{2}(0,0,0)=0, \quad a_{5}(0,0,0)=0 .
$$

Defining

$$
V:=\left(\sqrt{\alpha \kappa \delta} u_{x}, u_{t}, \theta, q\right)^{\prime}
$$

we obtain

$$
A^{0} V_{t}+A^{1} V_{x}+B V=\widetilde{F}\left(V, V_{x}\right), \quad V(0)=V_{0}:=\left(\sqrt{\alpha \kappa \delta} u_{0, x}, u_{1}, \theta_{0}, q_{0}\right)^{\prime}
$$

where

$$
\begin{gathered}
A^{0}:=\left(\begin{array}{cccc}
1 & 0 & 0 & 0 \\
0 & \kappa \delta & 0 & 0 \\
0 & 0 & \beta \kappa & 0 \\
0 & 0 & 0 & \beta \gamma \tau_{0}
\end{array}\right), \quad A^{1}:=\left(\begin{array}{cccc}
0 & -\sqrt{\alpha \kappa \delta} & 0 & 0 \\
-\sqrt{\alpha \kappa \delta} & 0 & \beta \kappa \delta & 0 \\
0 & \beta \kappa \delta & 0 & \beta \kappa \gamma \\
0 & 0 & \beta \kappa \gamma & 0
\end{array}\right) \\
B \\
:=\left(\begin{array}{cccc}
0 & 0 & 0 & 0 \\
0 & 0 & 0 & 0 \\
0 & 0 & 0 & 0 \\
0 & 0 & 0 & \beta \gamma
\end{array}\right), \quad \widetilde{F}:=\left(\begin{array}{c}
0 \\
\kappa \delta h_{1} \\
\beta \kappa h_{2} \\
\beta \gamma h_{3}
\end{array}\right)
\end{gathered}
$$

We have from (4.4) - (4.9)

$$
\widetilde{F}(0,0)=0, \quad \widetilde{F}_{\left(V, V_{x}\right)}(0,0)=0
$$

The linearized system, i.e. for $\widetilde{F}=0$, is solved by

$$
V(t)=e^{t R} V_{0}
$$

where

$$
R:=-\left(A^{0}\right)^{-1}\left(A^{1} \partial_{x}+B\right)
$$

generates a $C_{0}$-semigroup on $D(R):=\left(W^{1,2}(\mathbb{R})\right)^{4} \subset\left(L^{2}(\mathbb{R})\right)^{4}$. Then the solution to (4.10) is represented in general by

$$
V(t)=e^{t R} V_{0}+\int_{0}^{t} e^{(t-r) R} F\left(V, V_{x}\right)(r) d r
$$

where

$$
F:=\left(A^{0}\right)^{-1} \widetilde{F}
$$

The asymptotic behavior of the $L^{2}$-norm of the solution to the linearized problem is described by

Lemma 4.2 Let $F=0$. Then there are constants $c, c_{1}>0$ such that for all $t \geq 0$ : 
(i)

$$
\left\|\partial_{x}^{l} V(t)\right\|_{2} \leq c\left\{e^{-c_{1} t}\left\|\partial_{x}^{l} V_{0}\right\|_{2}+(1+t)^{-\frac{2 l+1}{4}}\left\|V_{0}\right\|_{1}\right\} \quad(l=0,1)
$$

(ii)

$$
\left\|\partial_{t} V(t)\right\|_{2} \leq c\left\{e^{-c_{1} t}\left\|V_{0}\right\|_{1,2}+(1+t)^{-\frac{3}{4}}\left\|V_{0}\right\|_{1}\right\}
$$

(iii)

$$
\left\|V_{4}(t)\right\|_{2} \leq c\left\{e^{-c_{1} t}\left\|V_{0}\right\|_{1,2}+(1+t)^{-\frac{3}{4}}\left\|V_{0}\right\|_{1}\right\}
$$

This lemma follows from the results of Yang \& Wang [24], in particular from the expansions of the characteristic values; cp. similar results for classical thermoelasticity in [8, Thm.3.6], going back to Kawashima [11].

The proofs of the $L^{2}$-decay results in classical nonlinear thermoelasticity (Fourier's law) repeatedly used the divergence forms of the differential equations arising from the balance law for the momentum (1.1) and for the energy (1.2). The same structure is not present in Cattaneo's law. Therefore, we restrict ourselves to a special case, namely we assume that $\tau$ and $k$ are constant functions,

$$
\tau \equiv \tau_{0}, \quad k \equiv \kappa
$$

that is, Cattaneo's law is the linear one:

$$
\tau_{0} q_{x}+q+\kappa \theta_{x}=0
$$

This assumption implies that in (4.3)

$$
h_{3}=0
$$

and for the remaining nonlinearities $h_{1}, h_{2}$ in (4.1), (4.2) we are able to carry over the considerations from classical thermoelasticity. We shall also exploit that the free energy $\psi$ and the internal energy have the form

$$
\begin{aligned}
\psi\left(u_{x}, \theta, q\right) & =\psi_{0}\left(u_{x}, \theta\right)+\psi_{1}\left(u_{x}, \theta\right) q^{2} \\
e\left(u_{x}, \theta, q\right) & =e_{0}\left(u_{x}, \theta\right)+e_{1}\left(u_{x}, \theta\right) q^{2}
\end{aligned}
$$

with suitable $\psi_{0}, \psi_{1}, e_{0}, e_{1}$, see [13]. Then we shall prove

Theorem 4.3 Let $V_{0} \in W^{3,2}(\mathbb{R}) \cap L^{1}(\mathbb{R})$, and $m_{0}:=\left\|V_{0}\right\|_{3,2}+\left\|V_{0}\right\|_{1}$. For sufficiently small $m_{0}$ there is $c>0$ such that for $t \geq 0$, the solution $V$ to (4.10) satisfies

$$
\|V(t)\|_{1,2} \leq c(1+t)^{-\frac{1}{4}} \cdot m_{o}
$$

Proof: With

$$
V=\left(\sqrt{\alpha \kappa \delta} u_{x}, u_{t}, \theta, q\right)
$$

we have

$$
V_{t}-R V=F\left(V, V_{x}\right)
$$

and, by (4.16),

$$
F\left(V, V_{x}\right)=\left(0, h_{1}, h_{2}, 0\right) .
$$

The fact that $F$ vanishes quadratically in zero, cp. the next lemma, is not sufficient despite the linear decay behavior like that of a heat equation, because terms like " $V \cdot V_{x}$ " appear, cp. Zheng \& Chen [25]. Therefore, we shall rewrite the nonlinearities in divergence form in order to be able to exploit the better decay of derivatives in the later estimates for $\|V(t)\|_{2}$, a modification of the corresponding proof in classical thermoelasticity, cp. [8]. 
Lemma $4.4 \quad$ (i)

$$
h_{1}\left(V, V_{x}\right)=\left(S\left(u_{x}, \theta, q\right)-\alpha u_{x}+\beta \theta\right)_{x} \equiv\left(h_{11}(V)\right)_{x}
$$

(ii)

$$
h_{2}\left(V, V_{x}\right)=\left(\left[\theta+\delta u_{x}-\gamma\left\{\varepsilon-\varepsilon(0,0,0)+\frac{u_{t}^{2}}{2}\right\}\right]\right)_{t}+\gamma\left(S u_{t}\right)_{x} \equiv\left(h_{21}(V)\right)_{t}+\left(h_{22}(V)\right)_{x}
$$

(iii)

$$
\left|h_{11}(V)\right|,\left|h_{21}(V)\right|,\left|h_{22}(V)\right| \leq c|V|^{2}
$$

(iv)

$$
\left|h_{1}\left(V, V_{x}\right)\right|,\left|h_{2}\left(V, V_{x}\right)\right| \leq c\left(|V|^{2}+\left|V_{x}\right|^{2}\right) \quad(\text { near } V=0)
$$

Proof: For (i) compare (4.6). For (ii) we first have

$$
\left(\varepsilon+\frac{u_{t}^{2}}{2}\right)_{t}=\left(S u_{t}\right)_{x}-q_{x}
$$

which is, using (1.1), equivalent to (1.2).

It follows

$$
\begin{aligned}
\theta_{t}+\delta u_{x t}+\gamma q_{x} & =\theta_{t}+\delta u_{x t}+\gamma q_{x}+\gamma\left\{\left(S u_{t}-q\right)_{x}-\left(\varepsilon+\frac{u_{t}^{2}}{2}\right)_{t}\right\} \\
& =\left(\theta+\delta u_{x}-\gamma\left\{\varepsilon-\varepsilon(0,0,0)+\frac{u_{t}^{2}}{2}\right\}\right)_{t}+\gamma\left(S u_{t}\right)_{x}
\end{aligned}
$$

which yields (ii) (cp.(4.2)).

$$
\left|h_{11}(V)\right|, \quad\left|h_{22}(V)\right| \leq c|V|^{2}
$$

follows immediately from the definition of $h_{11}, h_{22}$ and from (4.4) and $S(0,0,0)=0$ which is assumed without loss of generality.

The estimates (iv) also are immediate consequences of (4.4) - (4.7), so it remains to investigate $h_{21}(V)$.

$$
\begin{aligned}
h_{21}(V) & =\theta+\delta u_{x}-\gamma\left\{\varepsilon-\varepsilon(0,0,0)+\frac{u_{t}^{2}}{2}\right\} \\
& =\theta+\delta u_{x}-\gamma\left\{\varepsilon_{u_{x}}(0,0,0) u_{x}+\varepsilon_{\theta}(0,0,0) \theta+\varepsilon_{q}(0,0,0) q\right\}+\mathcal{O}\left(u_{x}^{2}+\theta^{2}+q^{2}+u_{t}^{2}\right)
\end{aligned}
$$

Observing

$$
\begin{aligned}
\varepsilon_{u_{x}}=\psi_{u_{x}}+\left(\theta+T_{0}\right) \eta_{u_{x}} & =S+\left(\theta+T_{0}\right)\left(-S_{\theta}\right), \\
\varepsilon_{\theta}=\psi_{\theta}+\eta+\left(\theta+T_{0}\right) \eta_{\theta} & =-\left(\theta+T_{0}\right) \psi_{\theta \theta}
\end{aligned}
$$

as well as (4.18) and (4.4), (4.5) we obtain

$$
\begin{aligned}
\gamma \varepsilon_{u_{x}}(0,0,0) & =-\gamma T_{0} S_{\theta}(0,0,0)=\delta \\
\gamma \varepsilon_{\theta}(0,0,0) & =-\gamma T_{0} \psi_{\theta \theta}(0,0,0)=1 \\
\varepsilon_{q}(0,0,0) & =0
\end{aligned}
$$

hence

$$
h_{21}(V)=\mathcal{O}\left(u_{x}^{2}+\theta^{2}+q^{2}+u_{t}^{2}\right)=\mathcal{O}\left(|V|^{2}\right)
$$

This proves Lemma 4.4. Q.E.D.

Continuing the proof of Theorem 4.3 we notice that, as a consequence of Lemma 4.4, we may rewrite $F$ as

$$
F\left(V, V_{x}\right)=\left(0, h_{1}\left(V, V_{x}\right), h_{2}\left(V, V_{x}\right), 0\right)^{\prime}=\left(0,\left(h_{11}(V)\right)_{x},\left(h_{21}(V)\right)_{t}+\left(h_{22}(V)\right)_{x}, O\right)^{\prime} .
$$


Since $W: t \mapsto e^{(t-r) R}\left(0,0,\left(h_{21}(V(r))\right), 0\right)^{\prime}$ solves

$$
W_{t}-R W=0, W(r)=\left(0,0, h_{21}(V(r)), 0\right)^{\prime}
$$

we conclude

$$
\begin{aligned}
& e^{(t-r) R}\left(0,0,\left(h_{21}(V(r))\right)_{r}, 0\right)^{\prime} \\
&= \partial_{r}\left(e^{(t-r) R}\left(0,0,\left(h_{21}(V(r))\right), 0\right)^{\prime}\right)+\partial_{t}\left(e^{(t-r) R}\left(0,0,\left(h_{21}(V(r))\right), 0\right)^{\prime}\right) \\
&= \partial_{r}\left(e^{(t-r) R}\left(0,0,\left(h_{21}(V(r))\right), 0\right)^{\prime}\right)+R\left(e^{(t-r) R}\left(0,0,\left(h_{21}(V(r))\right), 0\right)^{\prime}\right) \\
&= \partial_{r}\left(e^{(t-r) R}\left(0,0,\left(h_{21}(V(r))\right), 0\right)^{\prime}\right)-\left(A^{0}\right)^{-1} A^{1} \partial_{x} e^{(t-r) R}\left(0,0,\left(h_{21}(V(r))\right), 0\right)^{\prime} \\
&-\left(A^{0}\right)^{-1} B e^{(t-r) R}\left(0,0,\left(h_{21}(V(r))\right), 0\right)^{\prime}
\end{aligned}
$$

By the representation (4.12) we get, using (4.20),

$$
\begin{aligned}
V(t)= & e^{t R} V_{0}+\int_{0}^{t}\left\{\partial_{x} e^{(t-r) R}\left(0, h_{11}, h_{22}, 0\right)^{\prime}\right. \\
& \left.-\left(A^{0}\right)^{-1} A^{1} \partial_{x} e^{(t-R)}\left(0,0, h_{21}, 0\right)^{\prime}-\left(A^{0}\right)^{-1} B e^{(t-r) R}\left(0,0, h_{21}, 0\right)^{\prime}\right\} d r \\
& +\left[e^{(t-r) R}\left(0,0, h_{21}(V(r)), 0\right)^{\prime}\right]_{r=0}^{r=t}
\end{aligned}
$$

This representation will be used to estimate $\|V(t)\|_{2}$ while the simpler one given in (4.12) will be sufficient to estimate $\left\|\partial_{x} V(t)\right\|_{2}$.

Let

$$
M(t):=\sup _{0 \leq r \leq t}(1+r)^{\frac{1}{4}}\|V(r)\|_{1,2}
$$

By (4.21) and Lemma 4.2 we obtain

$$
\begin{aligned}
\|V(t)\|_{2} \leq & c(1+t)^{-\frac{1}{4}}\left(\left\|V_{0}\right\|_{2}+\left\|V_{0}\right\|_{1}\right)+c \int_{0}^{t}\left\{e^{-c_{1}(t-r)}\left\|\left(h_{11}, h_{22}, h_{21}\right)\right\|_{1,2}\right. \\
& \left.+(1+t-r)^{-\frac{3}{4}}\left\|\left(h_{11}, h_{22}, h_{21}\right)\right\|_{1}\right\} d r+c\left\|h_{21}(V)\right\|_{2}
\end{aligned}
$$

$c, c_{1}, \ldots$ denoting positive constants neither depending on $t$ nor on $V_{0}$.

Since

$$
\begin{aligned}
\left\|\left(h_{11}, h_{22}, h_{21}\right)\right\|_{1,2}(r) & \leq c\|V\|_{1,2}\|V\|_{\infty}(r) \\
& \leq c\|V\|_{1,2}\|V\|_{1,2}(r) \\
& \leq c(1+r)^{-\frac{1}{4}} M(r)\left\|V_{0}\right\|_{2,2}
\end{aligned}
$$

the latter by Theorem 4.1, and since

$$
\left\|\left(h_{11}, h_{22}, h_{21}\right)\right\|_{1} \leq c\|V\|_{2}^{2} \leq c(1+r)^{-\frac{1}{2}} M^{2}(r)
$$


we get

$$
\begin{aligned}
\|V(t)\|_{2} \leq & c(1+t)^{-\frac{1}{4}} m_{0}+c \int_{0}^{t}\left\{e^{-c_{1}(t-r)}(1+r)^{-\frac{1}{4}} M(r) m_{0}\right. \\
& \left.+(1+t-r)^{-\frac{3}{4}}(1+r)^{-\frac{1}{2}} M^{2}(r)\right\} d r+c(1+t)^{-\frac{1}{4}} M(t) m_{0} \\
\leq & c(1+t)^{-\frac{1}{4}} m_{0}(1+M(t))+c(1+t)^{-\frac{1}{4}}\left\{m_{0} M(t)+M^{2}(t)\right\} \cdot J
\end{aligned}
$$

where

$$
J=J(t)=\int_{0}^{t} e^{-c_{1}(t-r)}(1+r)^{-\frac{1}{4}}(1+t)^{\frac{1}{4}} d r+\int_{0}^{t}(1+t-r)^{-\frac{3}{4}}(1+r)^{-\frac{1}{2}}(1+t)^{\frac{1}{4}} d r
$$

satisfies

$$
\sup _{t \geq 0} J(t)<\infty
$$

(see Lemma 7.4 in [14]),

hence

$$
\|V(t)\|_{2} \leq c(1+t)^{-\frac{1}{4}}\left\{m_{0}(1+M(t))+M^{2}(t)\right\}
$$

To estimate $\left\|\partial_{x} V(t)\right\|_{2}$ we use (4.12) directly and the Lemmas 4.2 and 4.4 to similarly conclude

$$
\begin{aligned}
\left\|\partial_{x} V(t)\right\|_{2} \leq & c(1+t)^{-\frac{3}{4}}\left(\left\|V_{0}\right\|_{1,2}+\left\|V_{0}\right\|_{1}\right)+c \int_{0}^{t}\left\{e^{-c_{1}(t-r)}\left\|\left(h_{1}, h_{2}\right)\right\|_{1,2}\right. \\
& \left.+(1+t-r)^{-\frac{3}{4}}\left\|\left(h_{1}, h_{2}\right)\right\|_{1}\right\} d r \\
\leq & c(1+t)^{-\frac{3}{4}} m_{0}+c \int_{0}^{t}\left\{e^{-c_{1}(t-r)}(1+r)^{-\frac{1}{4}} M(r) m_{0}\right. \\
& \left.+(1+t-r)^{-\frac{3}{4}}(1+r)^{-\frac{1}{2}} M^{2}(r)\right\} d r \\
\leq & c(1+t)^{-\frac{1}{4}}\left\{m_{0}(1+M(t))+M^{2}(t)\right\}
\end{aligned}
$$

where the term " $\left\|V_{x x}\right\|_{\infty}$ " in the estimate for $\left\|\left(h_{1}, h_{2}\right)\right\|_{1,2}$ produces $\left\|V_{0}\right\|_{3,2}$.

Combining (4.26), (4.27) we get

$$
M(t) \leq c_{2}\left\{m_{0}(1+M(t))+M^{2}(t)\right\}
$$

Choosing

$$
m_{0} \leq \frac{c_{2}}{2}
$$

we obtain

$$
M(t) \leq 2 c_{2} m_{0}+c_{2} M^{2}(t)
$$

Since

$$
M(0) \leq c m_{0}
$$

it is a standard argument (cp. e.g. [14]) to conclude that — for sufficiently small $m_{0}$ — we have

$$
M(t) \leq c_{3}
$$

( $c_{3}$ being the smallest zero of $\left.f(x)=c_{2} x^{2}-x+2 c_{2} m_{0}\right)$ and then

$$
\|V(t)\|_{1,2} \leq c_{4}(1+t)^{-\frac{1}{4}} m_{0}
$$


which proves Theorem 4.3.

Q.e.d.

Acknowledgments: The first author was partially supported in the DFG-project "Hyperbolic Thermoelasticity" (RA 504/3-1), and the second author was partially supported by a key project from the NSFC under the grant 10531020 and a project from NSFC under the grant 10676020.

\section{References}

[1] Carlson, D.E.: Linear thermoelasticity. Handbuch der Physik VIa/2 (1972), 297-346.

[2] Chandrasekharaiah, DS.: Hyperbolic thermoelasticity: a review of recent literature. Appl. Mech. Rev. 51 (1998), 705-729.

[3] Coleman, B.D., Hrusa, W.J. and Owen, D.R. : Stability of equilibrium for a nonlinear hyperbolic system describing heat propagation by second sound in solids. Arch. Rational Mech. Anal. 94 (1986), 267-289.

[4] Dafermos, C.M. and Hsiao, L.: Development of singularities in solutions of the equations of nonlinear thermoelasticity. Quart. Appl. Math. 44 (1986), 463-474.

[5] Hansen, I.: Lebensdauer von klassischen Lösungen nichtlinearer Thermoelastizitätsgleichungen. Diploma thesis, University of Bonn (1994).

[6] Hrusa, W.J. and Messaoudi, S.A.: On formation of singularities in one-dimensional nonlinear thermoelasticity. Arch. Rational Mech. Anal. 111 (1990), 135-151.

[7] Hughes, T.J.R., Kato, T. and Marsden, J.E.: Well-posed quasi-linear second-order hyperbolic systems with applications to nonlinear elastodynamics and general realitvity. Arch. Rational Mech. Anal. 63 (1977), 273-294.

[8] Jiang, S. and Racke, R.: Evolution equations in thermoelasticity. $\pi$ Monographs Surveys Pure Appl. Math. 112, Chapman \& Hall/CRC, Boca Raton (2000).

[9] Kato, T.: The Cauchy problem for quasi-linear symmetric hyperbolic systems. Arch. Rational Mech. Anal. 58 (1975), 181-205.

[10] Kato, T.: Quasi-linear equations of evolution, with applications to partial differential equations. In: "Spectral theory and differential equations"; ed.: W.N. Everitt. Lec. Notes Math. 448 (1975), $24-70$.

[11] Kawashima, S.: Systems of a hyperbolic-parabolic composite type, with applications to the equations of magnetohydrodynamics. Thesis, Kyoto University (1983).

[12] Messaoudi, S.A. and Said-Houari, B.: Exponential stability in one-dimensional non-linear thermoelasticity with second sound. Math. Meth. Appl. Sci. 28 (2005), 205-232.

[13] Öncü, T.S. and Moodie, T.B.: On the constitutive relations for second sound in elastic solids. Arch. Rational Mech. Anal. 121 (1992), 87-99.

[14] Racke, R: Lectures on nonlinear evolution equations. Initial value problems. Aspects of Mathematics E19. Friedr. Vieweg \& Sohn, Braunschweig/Wiesbaden (1992).

[15] Racke, R.: Thermoelasticity with second sound - exponential stability in linear and non-linear 1-d. Math. Meth. Appl. Sci. 25 (2002), 409-441. 
[16] Racke, R. and Wang, Y.-G.: Propagation of singularities in one-dimensional thermoelasticity. $J$. Math. Anal. Appl. 223 (1998), 216-247.

[17] Racke, R. and Wang, Y.-G.: Asymptotic behavior of discontinuous solutions to thermoelastic systems with second sound. J. Anal. Appl. 24 (2005), 117-135.

[18] Racke, R. and Wang, Y.-G.: Propagation of strong singularities in 3-d thermoelasticity with second sound. Konstanzer Schriften Math. Inf. (2006).

[19] Tarabek, M.A.: On the existence of smooth solutions in one-dimensional nonlinear thermoelasticity with second sound. Quart. Appl. Math. 50 (1992), 727-742.

[20] Taylor, M.E.: Pseudodifferential operators and nonlinear PDE. Progress Math. 100, Birkhäuser, Boston (1991).

[21] Wang, W. and Z. Wang: The pointwise estimates to solutions for 1-dimensional linear thermoelastic systems with second sound. J. Math. Anal. Appl. 326 (2007), 1061-1075.

[22] Wang, Y.-G.: Microlocal analysis in nonlinear thermoelasticity. Nonlinear Analysis, T.M.A., 54 (2003), 683-705.

[23] Wang, Y.-G. and Yang, L.: $L^{p}-L^{q}$ decay estimates for Cauchy problems of linear thermoelastic systems with second sound in 3-d. Proc. Roy. Soc. Edinburgh, Sec. A, 136 (2006), 189-207.

[24] Yang, L. and Wang, Y.-G.: $L^{p}-L^{q}$ decay estimates for the Cauchy problem of linear thermoelastic systems with second sound in one space. Quart. Appl. Math. 64 (2006), 1-15.

[25] Zheng, S. and Chen, Y.: Global existence for nonlinear parabolic equations. Chin. Ann. Math. 7B (1986), 57-73.

Reinhard Racke, Department of Mathematics and Statistics, University of Konstanz, 78457 Konstanz, Germany, reinhard.racke@uni-konstanz.de

Ya-Guang Wang, Department of Mathematics, Shanghai Jiao Tong University, Shanghai 200240, P.R. China, ygwang@sjtu.edu.cn 